\title{
El uso didáctico del diablo cartesiano en la formación inicial de maestros*
}

\section{The educational use of Cartesian devil in the initial training of teachers}

\section{J. Carrasquer ${ }^{(1)}$, A. Ponz ${ }^{(1)}$, M. Talavera ${ }^{(2)}$, B. Carrasquer ${ }^{(3)}$ y M.V. Álvarez ${ }^{(1)}$}

(1) Dpto. de Didáctica de las C. E. Grupo Beagle. Universidad de Zaragoza (2) Dpto. de Didáctica de las C. E. y Sociales. Universidad de Valencia (3) C. Invest. de Recursos y Consumos Energéticos. Universidad de Zaragoza

Resumen: La relación didáctica entre profesorado y estudiante es esencial para alcanzar que éste construya su saber a partir de una transposición de los conocimientos. El primero debe plantear qué propuesta metodológica es la apropiada para que el alumnado alcance las competencias previstas. Los contenidos transpuestos no han de contradecir sustancialmente los planteamientos científicos que explican los fenómenos, independientemente del nivel educativo. El trabajo que se presenta propone la utilización de textos históricos para implicar al alumnado en la comprensión de los fenómenos físicosbiológicos a partir de actividades prácticas similares a las que condujeron a interpretar la función de la vejiga natatoria y paralelamente a la difusión del diablillo cartesiano, vinculado con la construcción de los primeros termoscopios.

Palabras clave: Diablo cartesiano, vejiga natatoria, utilización didáctica, historia de la ciencia, estrategias de resolución históricas.

Abstract: The didactic relationship between teacher and student is essential for the achievement of students' knowledge from a transposition of their ideas. The teacher must consider what methodological proposal is appropriate with the objective of reaching the expected educational competences by the student. Independently of the educational level, the transposed contents must not substantially contradict scientific approaches that explain phenomena. This work proposes the use of historical texts to involve the students in understanding the physico-biological phenomena from practical activities. These activities should be similar to those that led to interpret the function of the swim bladder and in parallel to the diffusion of Cartesian devil, linked to the first building thermoscopes.

Keywords: Cartesian devil, air bladder, educational use, history of science, historical resolution strategies.

(Fecha de recepción: abril, 2015, y de aceptación: marzo, 2016)

DOI: 10.7203/DCES.31.5157

* J. Carrasquer, A. Ponz y M. V. Álvarez pertenecen al Grupo Beagle de Investigación Aplicada en Didáctica de Ciencias Naturales, está financiado por el Gobierno de Aragón y el Fondo Social Europeo. Este trabajo es consecuencia del Proyecto de la Convocatoria de Innovación Docente 2013/14 de la Universidad de Zaragoza, PIIDUZ_13_538, en el que también participó la profesora M. Talavera. 


\section{Introducción}

El diablo cartesiano (a partir de ahora DC), pequeño buzo o ludión ha sido una herramienta didáctica y de entretenimiento desde el momento de la descripción de su funcionamiento junto con otra serie de juegos planteados por los Medici para seleccionar al sucesor de Torricelli al frente de la Accademia del Cimento de Florencia. En este trabajo se utiliza la historia de la ciencia y en concreto determinados fragmentos literarios donde los científicos protagonistas de los acontecimientos sugieren experiencias para demostrar sus planteamientos. A partir de su lectura y la tutorización histórica, se pretende que los maestros de Educación Primaria en formación alcancen un aprendizaje cognitivo y experimental que les permita ser capaces de diseñar en el futuro estrategias de aprendizaje para sus estudiantes.

Posteriormente se evaluará la comprensión del fenómeno físico trabajado mediante incorporación de una nueva variable, la corriente eléctrica.

Los resultados que aquí se presentan son una parte significativa de los obtenidos en una investigación más amplia (Proyecto de Innovación Docente e Investigación Educativa de la Universidad de Zaragoza 2014, PIIDUZ_13_538) y en concreto, los referentes a una actividad de evaluación de resultados, realizada incorporando la nueva variable, con el objetivo de comprobar la significación del aprendizaje de parte de los contenidos trabajados en el proyecto.

\section{Antecedentes}

El uso del DC tiene un amplio historial en el ámbito de la educación y surge nada más realizarse su primera descripción pública por Magiotti (1648). En la actualidad se utiliza con diversas modificaciones en todos los niveles educativos, adaptando las metodologías al nivel de los estudiantes.

Stoy (1784, 816-820), en Nuremberg, incluye al DC entre las actividades de física experimental que recomienda para trabajar con los jóvenes.

También se utiliza como una actividad didáctica en las escuelas de Francia por encargo del Conseil de l'Instruction Publique (1834, 11). Bary (1838, 173174) incluye al DC en su libro sobre nuevos problemas de física planteados en el Concurso general de física y química del Estado a partir del año 1805, una vez creada la estructura de lycées en Francia.

Héraud (1884, 241-242) también introduce el problema del funcionamiento del DC en su libro sobre juegos y recreaciones científicas. Asímismo Tom Tit, pseudónimo de Arthur Good $(1890,79-80)$ en su libro sobre ciencia divertida.

Galopin en su curso de física para candidatos a incorporarse a la Marina del Estado o comercial, también incluye la mencionada experiencia (Galopin, 1914, 75). 
Hoy se encuentra en las universidades con un objetivo científico o de aprendizaje para futuros formadores (Bürger, 1995; Güémez, Fiolhais y Fiolhais, 2003; Güémez, 2004; De Luca y Ganci, 2011; Élie, 2012).

Entre las investigaciones que se han llevado a cabo para analizar el uso didáctico del DC, se citan las de Frazier (1995; 2004), Adler (1997), Furió, Payá y Valdés (2005), Sanjosé y Torres (2010), Lozano (2012), Torres (2013), Sappington y Taylor (2014).

En estos trabajos, de forma general, se estudian las explicaciones que los estudiantes dan a los hechos observados. Si se quiere que el alumnado sea competente en distinguir entre la magia y la ciencia, entre el dogmatismo y las afirmaciones basadas en evidencias, es necesario que sepan explicar los hechos observados a partir de la realización de comprobaciones que les permitan sacar conclusiones y dar explicaciones mediante un discurso coherente y basado en pruebas (Reyes y Garritz, 2009).

Respecto al interés de motivar a los estudiantes con actividades que sorprendan por la observación de un acontecimiento no esperado o para el que no encuentran una respuesta con sus conocimientos previos, Furió et al. (2005) proponen prácticas de laboratorio tradicionales para que los estudiantes, de entre quince y dieciocho años, lleven a cabo investigaciones. La propuesta de trabajo de los autores afirma:

"Los profesores solemos tener acceso a experiencias sencillas que son sus- ceptibles de interesar a los estudiantes por sus resultados sorprendentes, por la posibilidad que les brindan de poner en práctica su inventiva, etc., y que contribuyen decisivamente a un mejor aprendizaje." (Furió et al., 2005, 97).

Frazier (1995) utiliza una pregunta para comenzar el proceso de indagación de los estudiantes entre once y catorce años: de dónde proviene el nombre de buzo cartesiano. Ésta no encuentra respuesta por parte de los estudiantes, más allá de la intuitiva asignación de la autoría a Descartes. En el intento de responder a esta pregunta, el profesor, mediante una afirmación y desechando la intervención de Descartes dice a los estudiantes: "El buzo lleva el nombre de un gran filósofo porque nos hace pensar". Esta afirmación quedará patente a lo largo del trabajo desarrollado. El profesor hace un repaso exhaustivo a la bibliografía didáctica y a la omnipresencia del pequeño buzo en libros de actividades de laboratorio, en libros de texto y diversos proyectos de enseñanza de las ciencias. Clasifica las respuestas de sus estudiantes a la pregunta ipor qué se desplaza el aparato?, agrupándolas y sistematizándolas en cinco modelos (Frazier, 1995; 2004): Teoría del peso; del aire; de la presión-flujo; de la presión-fuerza y del volumen-desplazamiento.

Adler realiza su tesis de maestría sobre el DC (Adler, 1997). Su estudio se llevó a cabo con estudiantes, de entre diez y trece años, que estaban familia- 
rizados con la realización de experimentos. Para fundamentar su trabajo, repasa desde una perspectiva histórica, los autores que han estudiado el origen del aparato y su denominación, llegando también a la conclusión de que no existen pruebas de que Descartes interviniera en su invención. Afirma que durante muchos años se utilizó para explicar conceptos de física, pero que recientemente se utiliza más para que el alumnado piense, mediante enfoques constructivistas; utiliza la metodología del estudio de casos. Toma la categorización de teorías realizada por Frazier (1995) y añade una sexta: la forma de la botella. El autor concluye que si bien los jóvenes explicaron el motivo por el que el buzo se desplazaba verticalmente, ninguna de las tres parejas estudiadas desarrolló una teoría global, de aplicación más amplia. Sin embargo valora el estudio de casos positivamente por su utilidad didáctica y concretamente el trabajo con datos históricos y la metodología constructivista (Adler, 1997).

Siguiendo la propuesta de Furió et al. (2005), la tesis doctoral de Lozano (2012) se centra en la ciencia recreativa como herramienta motivadora para mejorar la adquisición de competencias argumentativas en estudiantes. Entre las actividades propuestas está el DC, para llevar a cabo con alumnado de trece-catorce años (Lozano, 2012, 147149). Los resultados obtenidos presentan modelos de los estudiantes similares a los de Frazier (1995), teorías presión-flujo-fuerza y teoría del peso que es la que al final es aceptada por los estudiantes (Lozano, 2012, 187-191; 250-256).

Asimismo la tesis doctoral de Torres (2013) utiliza el DC como experiencia que cumple las condiciones de sorprender a los estudiantes y para la que no encuentran una respuesta convincente que dé explicación al hecho observado. Los conocimientos previos obligan al planteamiento de preguntas y a realizar actividades de comprobación para contrastar sus hipótesis, que den respuesta a los interrogantes planteados por ellos mismos para posteriormente hacer predicciones. El autor se centra en métodos de estimulación y valoración de la calidad de las preguntas que realizan los estudiantes ante un hecho observado, sorprendente para sus modelos mentales. Pone de manifiesto la dificultad que existe para averiguar las representaciones mentales que utiliza una persona para comprender, el funcionamiento de un aparato, aun manipulándolo y solicitándole que explicite verbalmente o por escrito cuáles son sus explicaciones ante el hecho observado (Torres, 2013, 284). Por otra parte indica que:

"La posibilidad de manipular los dispositivos y no sólo visualizar su funcionamiento, genera mayor número de preguntas explicativas y menor número de preguntas predictivas. [..]." (Torres, 2013, 288).

En la misma línea argumentativa Sanjosé y Torres $(2010,169)$, hacen referencia a las investigaciones de Ferguson y Hegarty e inciden en el interés 
de que el estudiante aplique lo aprendido resolviendo otros planteamientos diferentes para apreciar si ha habido mejor aprendizaje al realizar actividades manipulativas, capaz de ser utilizado de manera más eficiente fuera del contexto de aprendizaje inicial.

Tal como se ha puesto de manifiesto hasta el momento, la utilización didáctica habitual del DC es para trabajar contenidos de física, si bien, también es posible su utilización en otras áreas de conocimiento, dadas las implicacio- nes sociales y culturales del aparato tal como se pone de manifiesto en el Cuadro I, en el que se referencian diversos ámbitos o hitos históricos relacionados con la historia de la ciencia en los que el DC de forma directa o indirecta puede estar relacionado. En el proyecto motivo de este trabajo se utiliza la historia del descubrimiento del DC como elemento motivador y ejemplificante desde diversos aspectos, siguiendo la línea de trabajos anteriores.

\section{Cuadro I. Posibles usos históricos del DC}

Fuente: elaboración propia

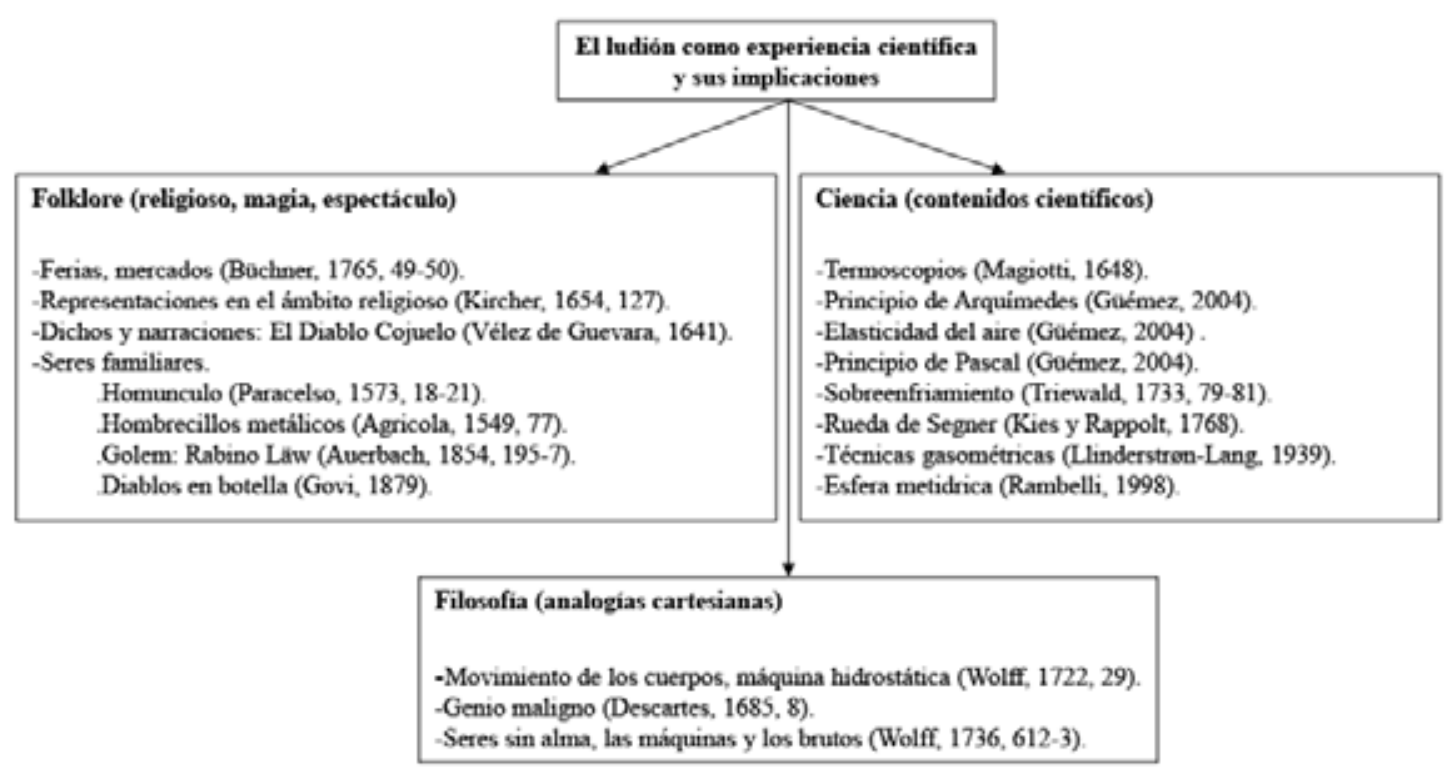

En los últimos años diversos informes de la Unión Europea (Rocard, 2006; Osborne y Dillon, 2008) ponen de manifiesto la escasa cultura científica de los ciudadanos europeos. Pero en concreto el informe Rocard, añade un aspecto más preocupante y es que los jóvenes europeos consideran la ciencia como difícil e irrelevante. Sin embargo la tecnología, está considerada como fundamental para la vida. Por ello autores como Van Aalderen-Smeets et al. 
(2012), consideran que es importante trabajar con los maestros en activo y los futuros docentes para que ellos mismos interioricen la importancia de la ciencia y tengan recursos suficientes para transmitir esas mismas sensaciones a su alumnado. Es necesario que los futuros maestros de Educación Primaria se formen adecuadamente en qué es la ciencia y su naturaleza, así como en la vinculación de las mismas con la tecnología (Guisasola y Morentin, 2007; Vázquez, Manassero y Ortiz, 2013).

\section{Los modelos físicos:}

\section{planteamiento cualitativo $y$ cuantitativo}

Cuando se habla del modelo físico de DC, los investigadores proponen distintos modelos en función de sus objetivos didácticos o sus planteamientos teóricos.

Los autores clásicos utilizan modelos de DC en los que la entrada y salida de agua es un orificio o tubo delgado. En estos casos lo habitual es considerar que el aparato se compone de la parte sólida, el agua y la burbuja de aire que está en su interior. Por ejemplo los representados Magiotti en la primera descripción del scherzo (Figura Ia), que años más tarde recibirá el nombre de $\mathrm{DC}$ dice:

“[...] Ciò avviene [...] perchè l'acqua, che non ammette compressione [...], essendo forzata, farà forza all'aria della caraffina, salendo per il collo di lei, [...]. Dunque la caraffina sarà più grave in specie, per l'acqua che v'è salita, e per l'aria che s'è condensata
[...] e così discenderà [...]." (Magiotti, 1648,14).

Libes también describe:

“[...] esferas de vidrio, y pequeñas figuras de esmalte suben o bajan de diferentes modos en una botella llena de agua, [...] estas pequeñas bolas estan compuestas de tres materias diferentes, á saber, agua, vidrio que es específicamente mas pesado que el agua, y aire que es mas ligero. [...]." (Libes, 1818, 130). Representadas en Figuras Ib y Ic.

Asimismo autores modernos dan esta misma explicación, Figura Ie:

"In this way the weight of the diver is given by the weights of the water inside and the weight of its glass portion, the weight of the air trapped inside being negligible." (De Luca y Ganci, 2011, 529).

En estos planteamientos anteriores, es de resaltar que varía la masa, pero no el volumen del aparato. Sin duda, hay que considerar que el empuje de flotación es menor al reducirse el tamaño de la burbuja.

Otros autores modernos consideran que el DC está compuesto exclusivamente por la pieza sólida y la burbuja de aire (Güemez, Fiolhais y Fiolhais, 2003). Es el modelo de la Figura Id. Se plantean DC con paredes rectas y un orificio en la parte inferior del mismo diámetro que el recipiente (tubo de ensayo): 
"Con objeto de simplificar los cálculos, el buzo de Descartes será un tubo de ensayo invertido, de vidrio, con una burbuja de aire en su interior, que flota sobre una superficie de agua contenida en un recipiente cerrado." (Güémez, 2004, 3).
$\mathrm{Al}$ igual que se ha hecho con el modelo anterior, en éste, contrariamente hay que resaltar que al descender el DC, varía el volumen, pero no la masa.

En la actualidad, este planteamiento es el utilizado en la práctica totalidad

\section{Figura I. Modelos de DC}

Fuente: elaboración propia

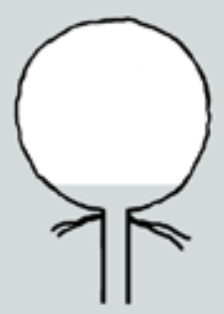

a

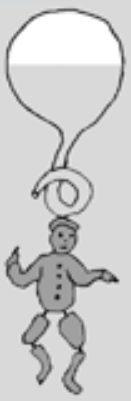

b

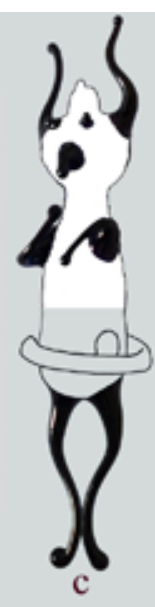

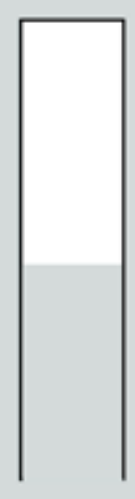

d

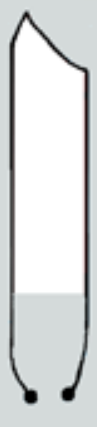

$\mathrm{e}$ de estudios cuantitativos del funcionamiento del DC, considerando como sus integrantes solamente la parte sólida y la gaseosa. De Luca y Ganci (2011) hacen notar que en estos casos tampoco se tiene en cuenta la adherencia del agua a las paredes de vidrio del diablillo. La simplificación de variables es necesaria, pero en este caso nos sugiere un interrogante. La transposición didáctica también recomienda la elección de algunas variables, que serán diferentes en función de cómo queramos abordar el problema con nuestro alumnado, con un planteamiento cualitativo o cuantitativo.
Para demostrar matemáticamente cómo funciona la física del DC no se considera la morfología clásica del DC en forma de figura hueca. Por lo tanto no tenemos en cuenta la masa del agua interna del DC y tampoco los aspectos físicos del movimiento de torbellino que provoca al salir por el tubo.

Cuando se trabaja desde el punto de vista cualitativo la explicación de los estudiantes basada en sus conocimientos previos, se centra mayoritariamente, tal como se ha puesto de manifiesto anteriormente, en la densidad, es decir en la relación entre la masa y el volumen. 
No hay que minusvalorar la dificultad para alcanzar un aprendizaje significativo de los conceptos flotar y hundirse (Álvarez y Bernal, 2000; Botero, 2010). En Maturano, Mazzitelli, Núñez y Pereira (2005) se puede comprobar la dificultad de los estudiantes para aplicar el Principio de Arquímedes correctamente, aun permitiendo a los estudiantes que busquen el enunciado e indicándoles que deben utilizarlo para explicar experiencias concretas realizadas por ellos.

Es interesante analizar los diferentes modelos de funcionamiento que presenta Frazier (1995). Tras la experimentación por parte de los estudian- tes para demostrar la pertinencia de sus cinco modelos iniciales, desechando algunos y acercando posturas entre otros, se resumen en los dos modelos que se indican en el Cuadro II.

Se interpreta que en la elección de un modelo interpretativo u otro, es relevante el formato de DC que se utilice para hacer la experiencia, además de los conocimientos previos de los estudiantes. En el caso que nos ocupa y teniendo en consideración los objetivos de aprendizaje se utiliza un planteamiento cualitativo, considerando que varía la masa del DC y no el volumen, es decir se considera el agua que está en el interior como parte del DC.

\section{Cuadro II. Modelos de funcionamiento del DC}

Fuente: interpretación de Frazier, (1995)

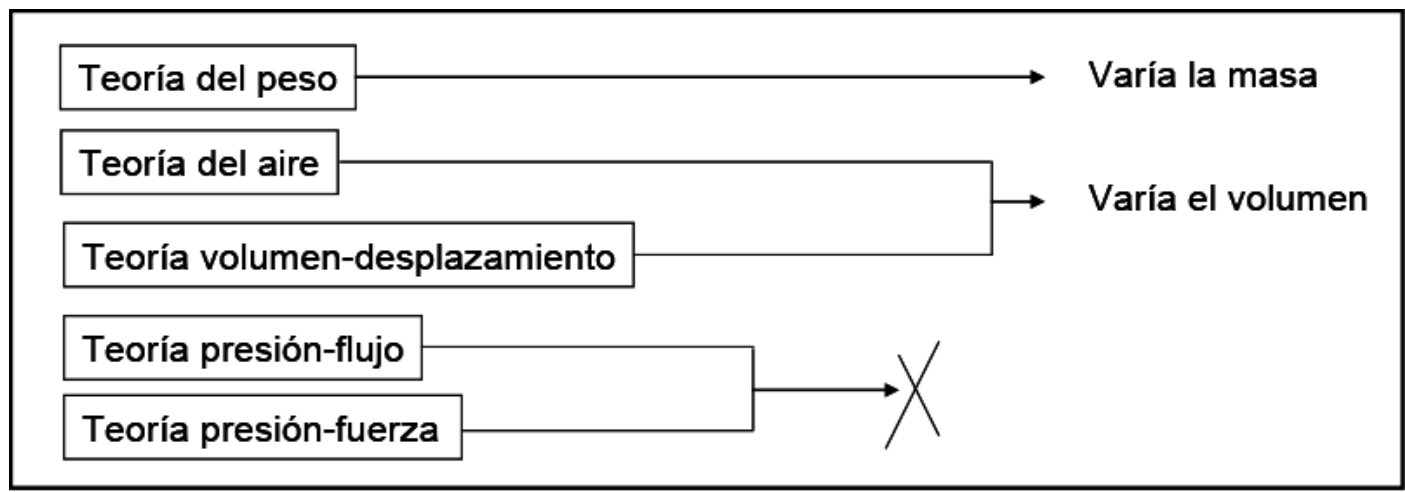

\section{Objetivos}

Los problemas habituales con los que se encuentran los estudiantes de Magisterio en las asignaturas de didácticas específicas y en concreto de Cien- cias Experimentales, están relacionados con las dificultades para llevar a cabo actividades experimentales, en concreto por la falta de unos mínimos conocimientos conceptuales, pero también por la falta de una actitud posi- 
tiva hacia la experimentación (Ponz, Carrasquer, Laguna, Esterán, Górriz, Álvarez y Carrasquer, 2015).

Los objetivos formativos que se han pretendido con la actividad global, de la que aquí se presenta sólo una parte tal como se ha indicado en la Introducción, son los de recordar o comprender determinados conocimientos de la física y el de acercar al alumnado de magisterio a la comprensión de aquellos aspectos que configuran el quehacer científico, es decir lo que se viene denominando como Naturaleza de la Ciencia y de la Tecnología, mediante la lectura y realización de experiencias realizadas para responder a preguntas. Asimismo familiarizar a los estudiantes con los textos históricos y su utilización como recurso didáctico ayudándose de herramientas de búsqueda bibliográfica, interpretación y selección de los mismos. También valorar las experimentaciones realizadas por los autores clásicos para responder sus preguntas, como válidas para responder las de los estudiantes.

La presentación de situaciones problemáticas, en las que habitualmente se ponen de manifiesto determinados obstáculos epistemológicos históricos, puede ayudar a los estudiantes a interpretar cómo se buscan y encuentran respuestas, y cómo el conocimiento científico es sustituido por nuevas interpretaciones de los acontecimientos observados. Para ello la experimentación tiene un valor imprescindible, que hay que potenciar desde los primeros años.

Como objetivo de investigación de la experiencia que aquí se presenta se pretende valorar el aprendizaje de los contenidos científicos implicados mediante actividades de aplicación en casos diferentes a los trabajados con las lecturas iniciales, introduciendo nuevas variables.

Los resultados presentados son los puestos de manifiesto con una actividad concreta en la que se introduce una nueva variable, la electricidad de una pila. Es decir, se propone a los estudiantes un problema ya conocido en un nuevo contexto. El objetivo de esta actividad es la de poner de manifiesto el aprendizaje conceptual alcanzado por los estudiantes de Magisterio con las actividades realizadas anteriormente.

Las actividades y los contenidos previstos son apropiados para integrar conocimientos científicos físicos y biológicos, tales como el principio de Arquímedes, el de Pascal, la elasticidad del aire, el funcionamiento de la vejiga natatoria de los peces, etc.

\section{Estrategias de resolución históricas}

Meavilla afirma que determinados problemas matemáticos han sido resueltos históricamente por procedimientos a los que denomina estrategias de resolución históricas y que pueden ser utilizadas como recurso didáctico. Asimismo define como tutorización histórica al "[...] conjunto de ayudas, ideas o sugerencias que se pueden ofrecer a aquellos que intentan resolverlo [...]" y que se obtienen de diversas estrategias extraídas del estudio de la Historia 
de la Ciencia (Meavilla, 2006, 84). Este planteamiento, aunque en el marco de las Matemáticas, se considera adecuado para utilizarse también en las Ciencias Experimentales.

Las actividades que aquí se presentan se llevaron a cabo con dos grupos de tercer curso de Magisterio Educación Primaria de la Facultad de Ciencias Sociales y Humanas de la Universidad de Zaragoza. Se considera pertinente indicar que estos estudiantes tuvieron una nota media de entrada en la titulación de 6,9 (PAU, 10+4 puntos posibles). Por otra parte, el $52 \%$ de ellos habían elegido asignaturas de ciencias experimentales en $4^{\circ}$ curso de Secundaria y el $19 \%$ cursaron bachilleres de estas materias. Por otra parte el Plan de Estudios de Magisterio Educación Primaria en la Universidad de Zaragoza tiene una carga docente de estas mismas materias de dos asignaturas de seis créditos cada una. Esta actividad se desarrolló en la segunda de ellas, con una asistencia media a clase de sesenta y nueve estudiantes (de los ciento dos matriculados en la asignatura) que se considera la muestra para el trabajo.

Tras una breve explicación sobre el ludión o DC, se pidió a los estudiantes universitarios que buscaran información acerca del funcionamiento del aparato, indicándoles que tendrían que explicarlo a niños/as de Educación Primaria del Colegio de Infantil y Primaria Anejas de la ciudad de Teruel, a los que paralelamente se había presentado el aparato para que realizaran preguntas acerca de su posible funcionamien- to (Galindo, 2014). Los estudiantes de Magisterio se documentaron acerca del DC y su relación analógica con la vejiga natatoria de los peces. Manipularon DC comerciales. En el curso anterior, una parte de ellos habían trabajado el aparato en la asignatura Didáctica del medio físico-químico. Veinticinco de estos estudiantes, en compañía de los profesores universitarios visitaron el Colegio Público Anejas para explicar a los estudiantes de Educación Primaria el funcionamiento del DC.

En esta fase inicial de la actividad se preguntó a los estudiantes acerca del funcionamiento de un DC (modelo construido con una paja de refresco, del profesor Hiromi O'Hara, según Danese, 2013). Ningún estudiante se refirió el principio de Arquímedes, ni al concepto de densidad para su explicación; los términos utilizados fueron principalmente: presión, masa, peso, observación de la entrada y salida de agua del interior de la paja y referencias a la existencia o no de aire en el cuello de la botella.

Paralelamente, el profesorado que ha participado en este proyecto seleccionó y adaptó los cuatro textos históricos que se indican a continuación:

- Primera lectura (Galileo, 1638, 69-70). Con esta actividad se pretendió que los estudiantes comprobaran prácticamente la dificultad-imposibilidad de mantener un cuerpo sólido en equilibrio estable a diversas alturas dentro del agua, considerando las variables que afectan. 
- Segunda lectura (Magiotti, 1648, 13-14). Reproduciendo la experiencia del autor se quiere que el alumnado compruebe, con la presión de la mano sobre el recipiente con agua, cómo sí se puede mantener a la altura que se desee en equilibrio el DC, y las variables que influyen. - Tercera lectura (A.I. y Boyle, 1675). Tal como describen el autor anónimo y Boyle se quiere demostrar experimentalmente cómo la presión de la columna de agua hace que una burbuja de gas (en este caso un globo) varía su tamaño.

- Cuarta lectura (Borelli, 1680, 335-6). Borelli plantea una analogía entre un pez y un cilindro y su émbolo. Se desarrolla en esta actividad calculando las diferentes densidades de la jeringuilla con el émbolo en tres posiciones.

Estas lecturas se seleccionaron entre otras por describir cada una de ellas un experimento que en su momento pudo ser fundamental para la comprensión física de varios problemas de esa época; entre otros, la elasticidad del aire, la existencia del vacío y la construcción de termoscopios. Se ha considerado adecuado para que el alumnado de Magisterio comprenda los fenómenos físicos implicados. Asimismo éstos son replicables en cualquier aula, y con pocos medios materiales, fácilmente accesibles para cualquier estudiante de magisterio o docente de Educación Primaria.

Las lecturas se facilitaron a los estudiantes universitarios. Cada grupo rea- lizó las experiencias que sugerían los textos a lo largo de seis horas de clase con la presencia de los profesores. Una vez realizadas las actividades se les pasó un cuestionario con preguntas abiertas acerca del desarrollo de las diversas actividades relacionadas con cada lectura (Los resultados de estas actividades quedan reflejados en la memoria del Proyecto de innovación citado en la introducción).

Finalizada la actividad anterior y a modo de evaluación de la comprensión de los contenidos de física trabajados se realizó otra actividad denominada, $E l$ Misterioso Sistema Cerrado, siguiendo la terminología de su autor, Penick.

Penick (1993) profesor en la Universidad de Iowa, describe las actividades realizadas por sus estudiantes en la construcción de ludiones. Entre la diversidad de variaciones del sistema físico que plantea a sus estudiantes, se refiere a una nueva versión de DC que describe, pero no desarrolla su puesta en práctica con el alumnado. Se refiere a ella como:

"[...] un sistema que utiliza una botella de vidrio de medio litro, de las que contienen licor (petaca), [...]. Conecte un extremo de un cable al terminal negativo de una batería de 1,5 voltios y sostenga el polo positivo contra la petaca de vidrio. [...] frotar el terminal positivo de la batería contra el vidrio [...]. Poco a poco, pero sin pausa, el pequeño tubo de ensayo comenzará a hundirse. Cuando el contacto con la batería o del cable cese, el buzo se 
elevará de nuevo a la parte superior. [...]" (Penick, 1993, 33).

Penick concluye que una vez que los estudiantes se despojan del entusiasmo científico pasajero al hacer las actividades, queda muy poco.

Berg (1993) de la Universidad de Wisconsin-Milwaukee, toma la propuesta de Penick y realiza la experiencia con sus estudiantes universitarios. Berg insiste en la importancia de la complejidad de los conceptos científicos y la necesidad de trabajarlos con calma y con la participación activa de los estudiantes en la experimentación. No es suficiente con dar una explicación al estudiante. Para demostrar este planteamiento describe su experiencia con el montaje del DC y la pila expuesto por Penick. Berg, después de que sus alumnos universitarios han construido sus propios DC, les plantea que describan cómo y porqué funciona el DC al frotar el polo positivo de la pila. Ante el nuevo planteamiento, los estudiantes no vinculan el funcionamiento a nada de lo aprendido anteriormente, se olvidan del efecto de la presión sobre la botella y también de que el vidrio es aislante. $\mathrm{El}$ autor continúa haciendo variaciones de la experiencia, por ejemplo haciendo llegar las vibraciones de distinta longitud de onda a otro DC.

Para comprobar la significación del aprendizaje con la actividad de lectura de textos históricos, mediante su aplicación en un nuevo problema, se realizó una experiencia similar a la planteada por Penick (1993) y Berg (1993) con die- cinueve pequeños grupos (3-5 estudiantes) de la asignatura indicada anteriormente (Figura II).

\section{Figura II \\ Estudiantes ante la réplica de la experiencia de Penick y Berg}

Fuente: elaboración propia

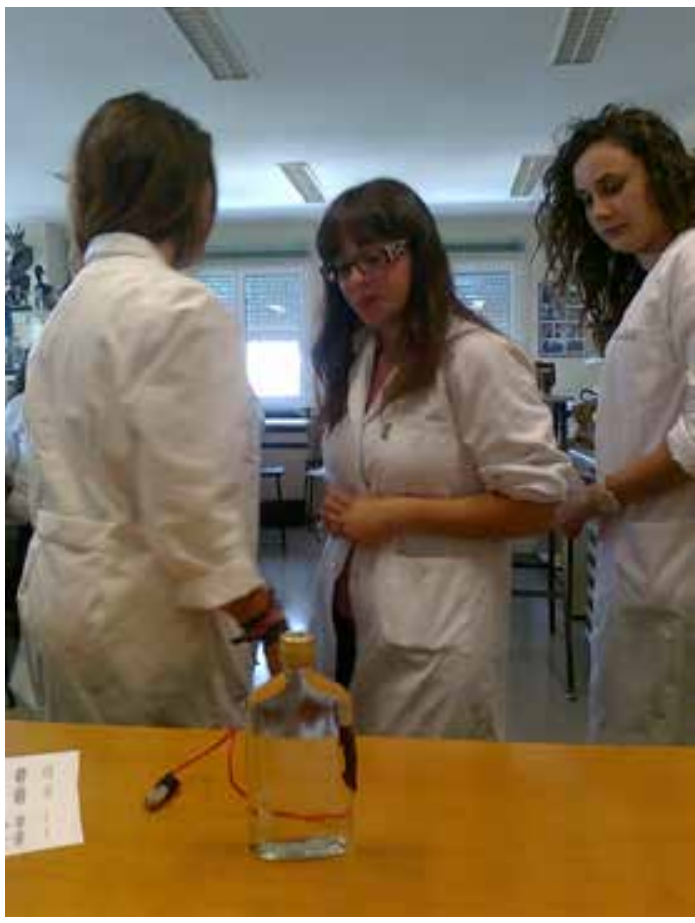

En el diseño de estas actividades es preciso tener presente las concepciones previas que utilizan los estudiantes para interpretar aquellos acontecimientos observados, dado que condicionan su aprendizaje. Para ello es fundamental la revisión previa de los abundantes estudios sobre estas ideas alternativas, y si fuese necesario la realización de nuevos estudios para lograr su detección. De esa manera se podría 
trabajar con modelos de pensamiento y reconducirlos a los que considere la comunidad científica en cada momento (Pozo y Gómez Crespo, 1998, 96-98). Sobre la necesidad de insistir en el trabajo docente, en aquellas ideas preconcebidas que se sabe tienen los estudiantes, insisten también Torres, Estela y Sanjosé $(2013,195)$.

Estas situaciones son de mayor relevancia cuando se trabajan contenidos fundamentales y reiterativos en los currícula, que son requisitos para la construcción de otros. Pero también en otros especialmente complejos, si se utilizan como herramienta analógica en el proceso de enseñanza. Este el caso del principio de Arquímedes, la vejiga de los gases de los peces y el diablillo cartesiano.

\section{Resultados}

El profesor/a realiza la experiencia de Penik con los estudiantes alrededor de una mesa; éstos inicialmente no realizan ninguna manipulación. El profesor da por terminada la actividad y pregunta si tienen alguna duda. Solicita a los estudiantes presentes que redacten lo que han visto y cuál es su explicación; son diecinueve grupos con un total de cincuenta y siete estudiantes que contestan la actividad (Anexo I). Cada uno de los grupos contesta por escrito a la pregunta, ¿Cuál es el motivo por el que el diablillo se mueve? Con las contestaciones realizadas por cada uno de los grupos se elabora una red sistémica que queda plasmada en el Cuadro I.
A continuación se transcriben las anotaciones que el profesor/a realiza a lo largo de esta experiencia con un grupo de clase y las consideraciones posteriores:

Una alumna (grupo 6) pregunta ¿has apretado la botella? El profesor dice no.

Los alumnos discuten y manipulan la botella. Se van para su sitio en el laboratorio. La alumna que había preguntado vuelve y frota la pila sobre la botella, observa que el DC no se mueve; seguidamente aprieta la botella y baja; la alumna se va sin decir nada.

Vienen otros grupos, frotan la pila con los dedos separada de la botella, frotan sobre el pantalón; aprietan el tapón metálico y aprietan la botella, todo ello de forma simultánea, de tal manera que provoca la incertidumbre de cuál es la variable que está afectando al movimiento del DC. (Seguramente estos grupos han oído los comentarios del grupo anterior).

Otros alumnos preguntan acerca de si el cable hace contacto con el vidrio, se les enseña que efectivamente está en contacto con el vidrio.

Otro alumno quiere confirmar, preguntando al profesor, si el vidrio es aislante; se le dice que sí.

Una alumna (grupo 6) después de retirarse a su sitio de trabajo, vuelve a la mesa donde está el montaje y comenta "hay algo que no me cuadra"; aprieta la botella, baja el DC y la estudiante vuelve a su sitio.

Otros estudiantes (grupo 9) vuelven a la mesa del montaje y comienzan a apre- 


\section{Cuadro III. \\ Síntesis de las causas por las que cada grupo indica que desciende el $\mathrm{DC}^{*}$}

Fuente: elaboración propia

\begin{tabular}{|c|c|c|c|c|c|c|}
\hline & & & Transmiten energía & & & 2 \\
\hline & vargas & Osilivas & Fricción de la pila & & & $\underline{13}$ \\
\hline & $\begin{array}{l}\text { Fricción polo } \\
\text { positivo }\end{array}$ & $\begin{array}{l}\text { Energía } \\
\text { positiva }\end{array}$ & $\begin{array}{l}\text { Aumento de la } \\
\text { temperatura }\end{array}$ & Aumento & & 1 \\
\hline & $\begin{array}{c}\text { Fricción } \\
\text { pila-botella }\end{array}$ & $\begin{array}{c}\text { Movimiento } \\
\text { de moléculas } \\
\text { del agua }\end{array}$ & A mayor fricción & $\begin{array}{l}\text { de la } \\
\text { presión }\end{array}$ & & 17 \\
\hline & Al frotar & $\begin{array}{l}\text { Sobrecarga } \\
\text { de energía }\end{array}$ & Disminuye la densid & ad del agua & & 5 \\
\hline & & & & & & 10 \\
\hline & Ene & gía & $\begin{array}{l}\text { Aumento del } \\
\text { volumen } \\
\text { del agua }\end{array}$ & $\begin{array}{c}\text { Compresión } \\
\text { del aire }\end{array}$ & $\begin{array}{l}\text { Aumento } \\
\text { de la } \\
\text { densidad }\end{array}$ & 12 \\
\hline causas & & & Efecto energía $=$ efe & cto presión & del DC & 14 \\
\hline & & & Varía la densidad & del agua & $\begin{array}{l}\text { Movimiento } \\
\text { de partículas }\end{array}$ & $\underline{7}$ \\
\hline & Corr & ente & Menor densidad & lel agua & $\begin{array}{c}\text { Cambia el } \\
\text { volumen del } \\
\text { agua }\end{array}$ & $\underline{9}$ \\
\hline & & & Calienta el b & ote & $\begin{array}{l}\text { Aumento de } \\
\text { presión }\end{array}$ & 3 \\
\hline & Circ & aito & El agua se carga con & electrones & $\begin{array}{c}\text { Aumenta la } \\
\text { densidad del } \\
\text { agua }\end{array}$ & 4 \\
\hline & Hidr & lisis & Varía & la densidad & & $\underline{11}, \underline{18}$ \\
\hline & $\begin{array}{l}\text { El polo posit } \\
\text { atrae molécu }\end{array}$ & $\begin{array}{l}\text { vo de la pila } \\
\text { las positivas }\end{array}$ & $\begin{array}{l}\text { Repele moléculas } \\
\text { negativas del agu }\end{array}$ & El mo & $\begin{array}{l}\text { imiento crea } \\
\text { oresión }\end{array}$ & 16 \\
\hline Sin & $\begin{array}{r}\text { Por varia } \\
\text { tempe }\end{array}$ & $\begin{array}{l}\text { ión de la } \\
\text { atura }\end{array}$ & & & & $\underline{7}$ \\
\hline eléctricas & Por $\mathrm{p}$ & esión & & & $, 8, \underline{9}, \underline{11}, \underline{13}, 1$ & $\underline{18}, 19$ \\
\hline
\end{tabular}

*En subrayado se señalan los grupos que manifiestan varias opciones 
tar la botella (seguramente han oído el comentario de la alumna anterior).

Otro grupo (grupo 8) prueba a frotar la pila en el vidrio en la parte más estrecha de la botella y también luego en la parte más ancha; confirman que no se desplaza el DC y comentan que la botella es de cristal apretándola ligeramente; el DC no se mueve. Le piden al profesor que lo haga otra vez. Están dando por un hecho que la botella de vidrio no es en absoluto elástica.

Otro grupo (grupo 11) prueba a apretar con más fuerza y el DC desciende.

Se puede afirmar que los estudiantes aparentemente han entendido el funcionamiento y las variables físicas que influyen, después de haber estado trabajando con el DC durante el cuatrimestre. Con la nueva experiencia se introduce una variable, la electricidad, por lo que intentan encontrar una interpretación diferente que haga intervenir esta nueva variable, de la que desconocen sus propiedades, como se pone de manifiesto en las contestaciones, pero que conduzca a un aumento de la presión en la botella, a una variación de la densidad del agua, o a un aumento de la temperatura. Que son las variables trabajadas previamente.

También se observa el desconocimiento (al menos funcional) de la elasticidad del vidrio, porque a ningún estudiante se le ocurre. Por ejemplo, un alumno toma en sus manos la botella, frota el polo positivo de la pila sobre ella y observa que no hay movimiento del diablillo; no se le ocurre apretar la botella de vidrio. Parece ser que están acostumbrados a buscar interpretaciones, según las variables que en ese momento se les solicita, es decir no integran otras variables ya conocidas, o bien quedan excluidas por no considerarlas viables (elasticidad de la botella). La estudiante que hace la pregunta al profesor acerca de si está apretando la botella, probablemente la realiza porque el DC se ha desajustado en su equilibrio y el profesor se ve obligado a presionar de manera ostensible.

Aparecen ideas de electricidad positiva y electrones con una masa que es capaz de variar la densidad del medio. Algunos estudiantes preguntan acerca de si el contenido de la botella es agua o tiene alguna sustancia más, seguramente pensando en la hidrólisis, que luego indicarán en sus planteamientos.

Los estudiantes no están acostumbrados (predispuestos) a experimentar, pues la mayoría de los grupos no solicitan poder hacer la prueba ellos mismos y comprobar el funcionamiento.

Las ocasiones en que el DC al bajar se queda estable en el fondo, desconcierta más todavía a los observadores.

En concreto, solamente cuatro de los grupos $(21,1 \%)$ indican que es exclusivamente la variación de la presión la causante del movimiento del DC. El resto de los grupos responsabilizan a la electricidad en mayor o menor medida como responsable del movimiento.

Tres grupos (15,8\%) también mantienen a la presión como causante del movimiento, pero además incluyen a la electricidad como responsable de la variación de la densidad del agua y ésta 
debido a la electricidad, que también se incluye como un factor determinante.

También un grupo $(5,3 \%)$ piensa que es la variación de la temperatura el motivo, pero sin responsabilizar explícitamente a la electricidad de ello.

Catorce grupos $(73,7 \%)$ de forma explícita, piensan que el frotamiento de la pila en el exterior del recipiente de vidrio puede hacer mover el DC; las causas explicadas son variables, así como la concreción de ellas en los escritos. Son de destacar las respuestas de cuatro grupos $(21,1 \%)$ que responsabilizan a la electricidad del aumento de la presión dentro del recipiente, y siete $(36,9 \%)$ indican que la electricidad es causante de la variación de la densidad, en dos de los casos variándola, en otros dos disminuyéndola y en tres aumentándola. Esta variación de la presión se achaca a directamente a la electricidad, o bien a que ésta provoca la hidrólisis del agua o simplemente a un aumento del volumen.

\section{Conclusiones}

Es preciso considerar que el objetivo de aprendizaje fundamental de este proyecto es que los futuros graduados en Magisterio avancen en su comprensión acerca de la naturaleza de la ciencia. Se ha puesto de manifiesto que si bien pueden haber logrado avances en este aspecto, un inconveniente fundamental es su deficiencia en conocimientos científicos básicos, que debieran haber alcanzado en Educación Primaria y Secundaria y que no es posible los recuperen en un grado universitario con una distribución de carga docente como la actual de magisterio.

Las deficiencias en el conocimiento de contenidos conceptuales implicados en las actividades, en muchos de los estudiantes de estos grupos, hace compleja su posible voluntad de aprendizaje. Es muy difícil recuperar el tiempo no dedicado. Y por otra parte lo que parece más difícil lograr es el ánimo a experimentar, a comprobar las observaciones que ellos no manipulan.

Inicialmente, se pone en evidencia, al realizar las actividades con tutorización histórica, que hay aspectos que no habían comprendido en las lecciones magistrales, en sus consultas bibliográficas, ni en la preparación de sus explicaciones para los estudiantes de Educación Primaria; en este momento sí hay un avance en el aprendizaje. Posteriormente, con la actividad de Penick, nuevamente se pone de manifiesto que en otros contenidos no ha habido un aprendizaje significativo, ni interacción con otros conocimientos, en un porcentaje considerable de los estudiantes.

Sin embargo, lo más preocupante es su falta de actitud crítica ante algo que no entienden y van a tener que enseñar. Especialmente cuando se trata de conceptos algo más complejos como la densidad, lo cual pone de manifiesto la existencia de unos planes de estudio que intentan abarcar un excesivo número de contenidos, sin planificar previamente las relaciones entre ellos, dificultando, por tanto, los procesos cognitivos de estos estudiantes (Botero, 2010), en nuestro caso, futuros docentes. 
$\mathrm{Al}$ contrario de lo que indica Berg, la mayoría de estos estudiantes no parecen haberse olvidado de los aspectos que condicionaban el movimiento del DC, sino que intentan construir un modelo de funcionamiento que conduzca mediante la electricidad a las variaciones de presión, temperatura o densidad que condicionen la bajada y subida del DC.

Esto muestra la necesitad de planes de estudio mejor concebidos, en los que se tenga en cuenta la interrelación de los contenidos, tanto a nivel cognitivo como social e histórico, para un correcto desarrollo.

$\mathrm{Al}$ igual que indican Torres, Estela y Sanjosé $(2013,195)$ se aprecian una serie de conceptos o modelos del funcionamiento del sistema "claramente erróneas", lo que debiera ser un motivo de preocupación y además una llamada de atención para insistir en aplicar didácticamente toda esa gran cantidad de estudios, que fundamentalmente se generaron en los años finales del siglo $\mathrm{XX}$, acerca de ideas alternativas de los aprendices y que parece que no se tienen en consideración para trabajar insistentemente en los diversos niveles educativos. Especialmente preocupante es que estos planteamientos erróneos se produzcan y con semejante profundidad en los futuros formadores.

Según se pone de manifiesto en recientes trabajos referenciados en el texto (Ponz et al., 2015) la deficiencia de conocimientos en contenidos básicos de Ciencias Experimentales, en utilización del lenguaje oral y escrito, en operaciones matemáticas básicas, entre otros, puede condicionar un aprendizaje no funcional de múltiples acontecimientos percibidos en la vida cotidiana, lo que potencia unas explicaciones alternativas al conocimiento científico. $\mathrm{La}$ implementación de tiempo académico en la formación de maestros/as, presencial o no, para llevar a cabo actividades experimentales que podría ayudar a solucionar estos problemas y sin duda a mejorar la formación integral de los futuros maestros, mejorando su pensamiento crítico y una percepción de su entorno más acorde a los tiempos, de desarrollo científico y tecnológico, que determinan una gran parte de la realidad de la sociedad actual.

\section{Agradecimientos}

Este trabajo se realizó en el marco de la convocatoria de proyectos de Innovación Docente e Investigación Educativa de la Universidad de Zaragoza 2014, PIIDUZ_13_538, titulado El estudio de un caso histórico apropiado para comprender la naturaleza de la ciencia. El Grupo Beagle de Investigación de Didáctica de las Ciencias Naturales está financiado por el Gobierno de Aragón y el Fondo Social Europeo.

\section{Referencias bibliográficas}

AGRICOLA, G., (1549). De Animantibus subterraneis Liber. [s. 1.]: Froben. Recuperado de «goo.gl/tQfiqe»

A.I. y BOYLE, R. (1675). A conjecture concerning the bladders of Air That 
are Found is Fishes, communicated by A.I; And illustrated by an Experiment Suggested by the Honorable Robert Boyle. Philosophical Transactions, 10, 310-311. Recuperado de «http://rstl.royalsocietypublishing.org/content/10/112-122/310.full. pdf+html?sid=82d3f132-7847-4ef588bf-51a06d986173»

ADLER, D. (1997). Student observations and explanations of a physical phenomenon: The Cartesian Diver. (Tesis de maestría).The University of British Columbia, Vancouver. Recuperado de «https://circle.ubc.ca/handle/2429/6412»

ÁlVAREZ, V. M. y BERNAL, M. A. (2000). Explicaciones cotidianas y científicas sobre flotar y hundirse. Alambique Didáctica de las Ciencias Experimentales, 25, 55-65.

AUERBACH, B. (1854). Spinoza. Mannheim: Berlag von Bassermann \& Mathn. Recuperado de «http://gutenberg.spiegel.de/buch/spinoza-5182/1»

BARY, M. E. (1838). Nouveaux problèmes de Physique suivis des questions proposées au concours général depuis 1805 jusqu'a ce jour, dans les classes de Physique et de Chimie. París: Hachette. Recuperado de «goo.gl/ j6Tau6»

BERG, C. A. (1993). Another look at the Mysterious Closed System. The Science Teacher, 90(9), 44-48.

BORELLI, J. A. (1680). De Motu Animalium. (vol. 1). Roma: Bernabó.
Recuperado de «http://gallica.bnf.fr/ ark:/12148/bpt6k512312»

BOTERO, H. J. (2010). Una revisión del concepto de densidad: la implicación de los conceptos estructurantes en el proceso de enseñanza-aprendizaje. Revista de Educación y pensamiento. 17, 23-36.

BÜCHNER, G. H. (1765). Merkwürdige Beyträge zu dem Weltlauf der Gelehrten. Langensalza: J. Ch. Martini. Recuperado de «goo.gl/7OmXRa»

BÜRGER, W. (1995). Cartesianische Taucher. En W. Bürger, Der Paradoxe Eierkocher, Physikalische Spielereien aus Professor Bürgers Kabinett (pp. 132-145). Berlín: Birkhäuser Verlag.

CONSEIL DE L'INSTRUCTION PUBLIQUE (1834).Instruction surles Objets Nécessaires pou L'Exposition des notions Élémentaires des Sciences Naturalles dans les Écoles Primaries. Lausanne : Ducloux. Recuperado de «goo.gl/SvoTuC»

DANESE, B. (2013). Le Caraffine di Magiotti. Recuperado de «http:// www.reinventore.it/sala-profes sori/2013/12/le-caraffine-di-magiot$\mathrm{ti} / »$

DE LUCA, R. y GANCI, S. (2011). A lot of good physics in the Cartesiandiver. Physics Educations, 46(5), 528-532. Recuperado de «http://iopscience.iop. org/0031-9120/46/5/001»

ÉLIE, F. (2012). Le ludion: pas si ludique que ça!... . Recuperado de «http://fred. elie.free.fr» 
DESCARTES, R. (1685). Meditationes De Prima Philosophia. Amsterdam: Ex Typographia Blaviana. Recuperado de «http://gallica.bnf.fr/ark:/12148/ bpt6k57490k»

FRAZIER, R. (1995). A Philosophical Toy. Recuperado de «http://courses. education.illinois.edu/CI241-scienceSp95/resources/philoToy/philoToy. html»

FRAZIER, R. (2004). Ein philosophisches Spielzeug. En H. Schreier (Ed.), SINUS-Transfer Grundschule. (pp. 35-50). Recuperado de «http://www. sinus-transfer.uni-bayreuth.de/ fileadmin/MaterialienIPN/NaWi G2b_fuer_Web.pdf»

FURIÓ, C., PAYÁ, J. y VALDÉS, P. (2005). ¿Cuál es el papel del trabajo experimental en la educación científica? En D. Gil, B. Macedo, J. Martínez, C. Sifredo, P. Valdés, y A. Vilches (Eds.), Cómo promover el interés por la cultura científica. Una propuesta didáctica fundamentada para la educación científica de jóvenes de 15 a 18 años (pp. 81-102). Santiago: Oficina Regional de Educación para la América Latina y Caribe, OREALC/ UNESCO.

GALILEO, G. (1638). Discorsi e Dimostrazioni Matematiche intorno à due nuove scienze. Leiden: Elsevir. Recuperado de «goo.gl/Hl8n3g»

GALINDO, P. (2014, 12 de Febrero). El ludión llega a las aulas de la mano de profesores del Campus. Diario de Teruel, p. 16.
GALOPIN, J. (1914). Cours de Physique a l'usage des candidats aux brevets de mécaniciens de la Marine L"Etat et de la Marine du Commerce. París: Challamel. Recuperado de «http://gallica. bnf.fr/ark:/12148/bpt6k54820491»

GOOD, A. (1890). La Science Amusante. París: Larousse. Recuperado de «http://gallica.bnf.fr/ark:/12148/ bpt6k5406984m/f6.image»

GOVI, G., (1879). In che tempo e da chi siano stati inventati i Ludioni detti orinariamente: Diavoletti Cartesiani; Nota del socio Ordinario G. Govi. In Rendiconto dell'Academia delle Scienze Fisiche e Matematiche (Sezione delle Società Reale di Napoli). $\mathrm{N}^{\circ}$ 18, pp. 291-296.

GUÉMEZ, J. (2004). Buzo de Descartes (Cartesiandiver). Departamento de Física Aplicada, Universidad de Cantabria. 1-12. Recuperado de «http:// es.scribd.com/doc/123869249/Buzode-Descartes»

GUÉMEZ, J., FIOLHAIS, C, y FIOLHAIS, M. (2003). A Demostratio Apparatus for the Certesian Diver. The Physics Teacher, 41, 67-68. Recuperado de «http://www.loreto.unican. es/ACurriculum/TPTCartesianDiver. pdf»

GUISASOLA, J. y MORENTÍN, M. (2007). Comprenden la naturaleza de la ciencia los futuros maestros y maestras de Educación Primaria. Revista Electrónica de las Ciencias, 6(2), 246-262. Recuperado de «http:// reec.uvigo.es/» 
HÉRAUD, A. (1884). Jeux et récréations scientifiques. París: Baillière et Fils. Recuperado de «http://gallica.bnf.fr/ ark:/12148/bpt6k5774153b»

KIES, J. \& RAPPOLT, G. T. (1768). Dilucidationes Analyseos Finitorum Kaestnerianae. Tübingen: Sigmundi. Recuperado de «https://books.google. es/books?id=8r5KAAAAcAAJ\&print sec=frontcover\&hl=es\&source $=g$ s $_{-}$ ge_summary_r\&cad $=0 \# \mathrm{v}=$ onepage $\&$ $q \& f=$ false»

KIRCHER, A. (1654). Magnes sive de Arte Magnetica. ( $3^{\mathrm{a}}$ ed.) Roma: Vitalis Mascardi. Recuperado de «https:// books.google.es/books?id=pymMGN mnxzgC\&printsec=frontcover $\& h l=e s$ \&source=gbs_ge_summary_r\&cad $=0$ \#v=onepage\&q\&f=false»

LIBES, A. (1818). Tratado de Física completo y elemental. Barcelona: Brusi. Recuperado de «https://books.google. es/books?id=L38KYP6hzb8C\&print sec=frontcover\&hl=es\&source $=g$ gs $_{-}$ ge_summary_r\&cad $=0 \# \mathrm{v}=$ onepage $\&$ $q \& f=$ false»

LOZANO, O. R. (2012). La ciencia recreativa como herramienta para motivar y mejorar la adquisición de competencias argumentativas. (Tesis doctoral). Departamento de Didáctica de las Ciencias Experimentales y Sociales, Universidad de Valencia.

LLINDERSTRØM-LANG, K. (1937). Principle of the Cartesian Diver applied to Gasometric Technique. Nature, 140, 108.
MAGIOTTI, R. (1648), Renitenza certissima dell' Acqua alla Compressione, Dichiarata con varij scherzi, in occasione d' altri Problemi curiosi. Roma: F. Moneta. Disponible en: http:// www.internetculturale.it/jmms/iccuviewer/iccu.jsp?id=oai\%3Awww.imss. fi.it\%3A17\%3AFI0029\%3A367974\& mode $=$ all \&teca $=$ Museo+Galileo

MATURANO, C., MAZZITELLI, C., NÚÑEZ, G. y PEREIRA, R. (2005). Dificultades conceptuales y procedimentales en temas relacionados con la presión y los fluidos en equilibrio. Revista electrónica de Enseñanza de las Ciencias, 4, (2), s/p. Recuperado de «http://reec.uvigo.es/»

MEAVILLA, V. (2006). Problemas de persecución: tres ejemplos de tutorización histórica y algunas consideraciones de carácter didáctico. Eureka, Revista de la Licenciatura en Matemáticas Aplicadas, 21, 84-92. Recuperado de «http://www.uaq.mx/ingenieria/publicaciones/eureka/»

OSBORNE, J. y DILLON, J. (2008). Science education in Europe: Critical reflections (a report to the Nuffield Foundation). London: the Nuffield Foundation. Recuperado de «http:// www.pollen-europa.net/pollen dev/ Images Editor/Nuffield report.pdf»

PARACELSO, (1573). Summi Philosophi ac Medici, De Natura rerum libri Septem. Basileae: Pernam. Recuperado de «goo.gl/KYO0XO»

PENICK, J.E. (1993). The mysterious closed system. The Science Teacher, 60(2), 30-33. 
PONZ, A., CARRASQUER, J., LAGUNA, J.I., ESTERÁN, P., GÓRRIZ, M., ÁLVAREZ, M.V. Y CARRASQUER, B. (2015). Perfil del alumnado de los Grados de Magisterio en función de los estándares de aprendizaje evaluables de la LOMCE. En P. Membiela, N. Casado y M.I. Cebreiros (Eds.), La docencia universitaria: desafios $y$ perspectivas (en prensa). Ourense: Educación Editora.

POZO, I. y GÓMEZ CRESPO, M. A. (1998). Aprender y enseñar ciencia. Madrid: Morata.

RAMBELLI, F. (1998). La "Sfera metidrica". HDS Notizie, 9 (IV), 8-15. Recuperado de «http://www.hdsitalia.org/sites/www.hdsitalia.org/files/ documenti/hdsn09_giugno_1998.pdf»

REYES, F. M. y GARRITZ, A. (2009). El conocimiento pedagógico de la indagación científica del personal guía que impartirá actividades indagatorias de ciencia a profesores de la educación básica. X Congreso Nacional de investigación Educativa, Veracruz. Recuperado de «goo.gl/iK5dp4»

ROCARD, M. (2006). Informe sobre la enseñanza científica en Europa. Publicación electrónica. Recuperado de «http://www.ec.europa.eu/ research/science-society.»

SANJOSÉ, V. y TORRES, T. (2010). Preguntas de los estudiantes sobre dispositivos científicos. CiDd: II Congrés Internacional de Didàctiques 2010, (pp.1-7). Universidad de Girona, 3-6 de febrero.
SAPPINGTON, E. y TAYLOR, M. (2014). Hands-on Activity: Cartesian Diver. University of Houston. Recuperado de «goo.gl/EBt6G2»

STOY, J. S. (1784). Bilder Akademie fúr die Jugend. (vol. 2). Nuremberg: Sirischen Schristen. Recuperado de «goo.gl/XZNp9K»

TORRES, T. (2013). Preguntas de los estudiantes sobre dispositivos experimentales en distintas situaciones didácticas: Génesis y tipología. (Tesis doctoral). Universitat de València.

TORRES, T., ESTELA, B. y SANJOSÉ, V. (2013). Un estudio del contenido científico de las preguntas formuladas por estudiantes cuando intentan comprender dispositivos experimentales. Didáctica de las Ciencias Experimentales y Sociales. 27, 179-197. (Capítulo 9, Tesis doctoral, T. Torres, 2013).

TRIEWALD, M. (1733). A Letter from Mr. Triewald. Philosophical Transactions, (vol. 37, 1731-1732) pp. 79-81. Recuperado de «goo.gl/Tp8oay»

VAN AALDEREN-SMEETS, S. I., WALMA VAN DER MOLEN, J. H. y ASMA, L. J. F. (2012). Primary Teachers' Attitudes Toward Science: A New Theoretical Framework. Science Education, 96(1), 158-182.

VÁZQUEZ, A., MANASSERO, M. A. y ORTIZ, S. (2013). Análisis de materiales para la enseñanza de la naturaleza del conocimiento científico y tecnológico. Revista Electrónica de Enseñanza de las Ciencias, 12(2), 
243-268. Recuperado de «http://reec. uvigo.es/»

VÉLEZ DE GUEVARA, L. (1641). $E l$ Diablo Cojuelo. Madrid: Imprenta del Reyno. Recuperado de «goo. gl/0vmHUo»

WOLFF, Ch. (1722). Allerhand Nützliche Versuche, Dadurch Zu genauer Erkäntnis Der Natur und Kunst
Der Weg gebähnet wird, (vol. 2). Halle-Magdeburg: Rengerischen Buchhandlung. Recuperado de «goo. gl/43DFFS»

WOLFF, Ch. (1736). Theologia Naturalis, Methodo Scientifica Pertractata. (vol. 1). Frankfurt-Leipzig: Libraria Rengeriana. Recuperado de «goo.gl/ saIZqQ" 


\section{Anexo}

\section{Transcripciones de las explicaciones por grupos de la actividad el Misterioso Sistema Cerrado}

\section{Grupo 1}

A partir de la fricción del polo positivo de la pila con la botella de vidrio, crea energía positiva que se transmite al agua haciendo que aumente la temperatura y por lo tanto la presión aumenta y hace que baje la botellita.

Grupo 2

Creemos que la botella baja por las cargas positivas ejercidas por la pila. Al ser dos vidrios (botella y botellita) transmite una energía que hace que la botellita descienda.

\section{Grupo 3}

Explicación: A través de la corriente que le proporciona la pila, ésta calienta el bote y al aumentar de temperatura sube la presión y desciende la botella.

\section{Grupo 4}

Al colocar la pila tocando el frasco de cristal, se crea un circuito en el cual el agua que contiene se carga con electrones. Esto hace que el agua sea más densa y como consecuencia baje el frasco.

\section{Grupo 5}

Al frotar la pila con el vidrio se crea energía, y el cable es conductor de esta energía, lo que hace que aumente la temperatura del agua por lo que la densidad de esta baja, de esta manera la botellita del interior se hunde. Cuando hay sobrecarga de energía, la botellita se mantiene abajo hasta que el agua recupera la temperatura inicial.

\section{Grupo 6}

Creemos que el resultado de que el recipiente pequeño se sumerja, es debido a la presión que se ejerce en la botella, aunque la presión sea minima. Además creemos que la pila y el cable no tienen nada que ver con la presión, porque no está conduciendo ni variando ninguna variable.

\section{Grupo 7}

Hemos observado como un cuerpo se mueve dentro del recipiente de agua tapado. En dicho recipiente hay un cable adherido a la botella por un extremo y por el otro a una pila. Cuando dicha pila toca la botella de cristal, el cuerpo de dentro sube y baja. Creo que esto se debe a que la corriente de la pila hace que la densi- 
dad del agua varíe y se muevan las partículas que hay dentro del recipiente. Por otro lado, agarramos con la otra mano el recipiente. También podía deberse al calor que aplicamos a la botella al agarrarla, que hace que la temperatura del agua varíe y que por tanto, el cuerpo se mueva.

Grupo 8

La pila y el cable no condicionan nada. El hecho de que el frasquito baje es debido a la presión que haces con la mano al apretar la botella. Esto hace que aumente la presión y descienda. Si sueltas sube.

Grupo 9

Se varía la densidad del agua haciéndola menor que el ludión. Por lo tanto el ludión baja.

Conclusión: Se está cambiando el volumen que el agua ocupa en el recipiente. (La pila es una distracción). Realmente, lo que sucede es que se ejerce una presión en el recipiente que hace que varíe el volumen del recipiente.

Grupo 10

Experimento con pila, cable y botella de cristal llena de agua con una probeta. Pensamos que a través de la energía que transmite la conexión de la pila y el cable disminuye la densidad del agua y, por lo tanto, se iguale a la densidad del objeto. De esta manera, gracias a esa energía, el objeto flota, se hunde y se mueve.

Grupo 11

Al ser el agua un buen conductor de la electricidad al juntar la pila con el agua se produce la hidrólisis del agua alterando, así la densidad y provocando, como consecuencia, la bajada del ludión.

Al intentarlo nosotros mismos nos hemos dado cuenta que lo que hemos puesto arriba no influye porque el cable no está dentro de la botella. Por ello, solo hace falta presionar la botella para que el ludión baje.

Grupo 12

La pila a través del cristal ha transmitido energía y el agua al ser conductor de la electricidad ha cogido volumen, de tal modo que la probeta ha comprimido el aire, aumentando así su densidad. Por ello, al aumentar la densidad la probeta se hunde. 


\section{Grupo 13}

No tenemos claro a qué se debe pero pensamos que podría deberse a la presión ejercida sobre la botella con la mano y a la fricción de la pila, que está cargada positivamente.

\section{Grupo 14}

Al transmitir energía al interior de la botella, sucede el mismo efecto que al ejercer presión sobre ella. Por lo tanto, el ludión aumenta su densidad y provoca su hundimiento porque la densidad del ludión es mayor que la del agua.

\section{Grupo 15}

Pienso que, al ser una botella de plástico y no de otro material, al ejercer una presión sobre ésta, parte del agua que hay dentro del recipiente, entra dentro del ludión y hace que éste baje, más o menos dentro del recipiente.

Grupo 16

La pila al coger contacto con el recipiente de cristal, por su parte positiva, atrae las moléculas positivas y repele las moléculas negativas del agua. Al crear presión conforme se va moviendo la pila en pequeños círculos hace que el frasco del interior descienda.

\section{Grupo 17}

Partimos de la idea de que el agua es conductora de la electricidad. La fricción entre la pila y la botella de vidrio genera el movimiento de moléculas del agua contenidas en la botella. A mayor fricción, la presión del agua aumenta, lo que hace que el cuerpo sólido que se encuentra en el interior descienda.

\section{Grupo 18}

Ya que el agua conduce la electricidad, al juntar la pila con el agua se produce la hidrólisis del agua, por lo que altera su densidad provocando que el ludión baje. Nos hemos dado cuenta que el cable no está en contacto directo con la botella, así pues el ludión baja al ejercer presión sobre la botella, ya que ésta no es rígida y se puede presionar para modificar la altura del ludión.

\section{Grupo 19}

Tal y como hemos observado anteriormente utilizando junto al ludión un frasco de cristal, al hacer presión en los laterales de la botella este desciende, pudiendo adoptar la posición que queramos. La pila es una trampa para distraer. 
